\title{
Des milieux des uns aux discours des autres : à propos d'interactions médiatiques
}

\author{
Laurent Rouveyrol
}

\section{(2) OpenEdition}

\section{Journals}

Édition électronique

URL : http://journals.openedition.org/asp/853

DOI : $10.4000 /$ asp. 853

ISBN : 978-2-8218-0398-5

ISSN : 2108-6354

Éditeur

Groupe d'étude et de recherche en anglais de spécialité

Édition imprimée

Date de publication : 1 décembre 2004

Pagination : 43-59

ISSN : 1246-8185

\section{Référence électronique}

Laurent Rouveyrol, « Des milieux des uns aux discours des autres : à propos d'interactions

médiatiques », ASp [En ligne], 45-46 | 2004, mis en ligne le 02 mars 2010, consulté le 20 avril 2019.

URL : http://journals.openedition.org/asp/853 ; DOI : 10.4000/asp.853

Ce document a été généré automatiquement le 20 avril 2019.

Tous droits réservés 


\title{
Des milieux des uns aux discours des autres : à propos d'interactions médiatiques
}

\author{
Laurent Rouveyrol
}

\section{Introduction}

1 Les quelques énoncés qui précèdent sont tirés de l'émission de télévision Question Time, débat-panel phare de la $B B C$, mettant en scène des journalistes, des politiciens et les membres d'un public. Il paraît difficile à la lecture, de déterminer quel type de locuteur est à la source de chacun d'eux : s'agit-il d'un homme politique? d'un journaliste? La réponse est loin de s'imposer a priori. Nous allons donc tenter, dans cet article, de rendre compte de ce brouillage. Notre point de départ sera la définition de travail que donne Michel Petit (2002) des milieux professionnels, il s'agit d'un :

[...] espace social regroupant l'ensemble des membres de la société exerçant leur activité professionnelle (régulière, permettant de gagner sa vie) dans un même domaine socialement reconnu et présentant certaines caractéristiques d'organisation, de fonctionnement, etc. On parle souvent de milieux au pluriel (les milieux de la mode, les milieux politiques) ou de monde au singulier (le monde de la justice, le monde universitaire). (Petit 2002)

2 M. Petit prend soin de préciser que les milieux professionnels ne se réduisent pas à une profession :

En ce sens, le milieu professionnel ne se définit pas strictement par une profession (par exemple les milieux militaires, journalistiques). (Petit 2002)

3 La structure de l'émission et la nature des invités nous plongent au cœur du monde médiatique. Ses composants sont les milieux politique et journalistique, complétés par la dimension de la vie ordinaire, inhérente au processus de médiatisation: une interaction sur un plateau de télévision est diffusée vers de multiples récepteurs, dans un cadre domestique. 
4 N. Fairclough (1998) dira d'ailleurs que l'ordre du discours politique médiatisé est un " generic complex», formé à partir des ordres de discours de la politique, du monde des médias et de la vie quotidienne. Le politique et le journalistique sont donc liés très étroitement : que serait la politique sans médiatisation des idéologies? Que seraient les médias sans la possibilité de se faire l'écho, de relayer l'ordre politique du pays?

5 Nous allons nous intéresser plus particulièrement aux pratiques langagières des politiciens et des journalistes, pour montrer qu'elles ont tendance à se rapprocher dans et par l'interaction médiatique. Ces deux catégories d'acteurs font certes partie d'une communauté discursive bien distincte, poursuivant des buts professionnels spécifiques : le journaliste souhaite faire une émission de télévision intéressante qui séduise le plus grand nombre de téléspectateurs, le politicien tente d'assurer la promotion d'une idéologie pour se faire élire. On remarque déjà que ces deux communautés discursives ont des visées perlocutoires et incitent à une certaine forme d'action, d'inter-action. Les deux ordres ne se confondent donc pas mais on pourrait dire, en simplifiant, que par leur pratique discursive, les différents acteurs font de la même façon des choses différentes.

6 L'approche que nous allons adopter est résolument pragmatique : sont pris en compte les types de locuteurs dans un cadre interactionnel donné, les tâches cognitivo-discursives, les stratégies langagières qu'ils co-construisent, orientées vers les buts perlocutoires qu'ils s'assignent. Dans un premier temps, nous allons nous intéresser rapidement à l'aspect culturel de l'interaction politico-journalistique dans le cadre médiatique. Nous concentrerons ensuite notre attention sur les stratégies et pratiques langagières coconduites, afin d'identifier les types de rapprochements possibles. On retiendra particulièrement le phénomène des positionnements énonciatifs tels que l'opposition, le parallélisme ainsi que les stratégies de planification discursive. Chaque phénomène ne sera pas étudié per se mais dans la perspective où il marque un certain rapprochement.

\section{1. À propos de quelques données culturelles}

\subsection{De la Chambre au studio}

7 Tout d'abord, l'émission se nomme Question Time et renvoie à un épisode de la vie politique institutionnelle anglophone : les questions au gouvernement de la Chambre des communes. Le speaker de la BBC annonçant le programme de la soirée dira d'ailleurs : « and now, David Dimbleby is in the chair for Question Time ", consacrant ainsi le parallèle.

8 La structure même de l'émission du point de vue de l'organisation des débats, rappelle celle de la Chambre des communes. Les membres du plateau s'adressent au présentateur de l'émission ; les membres du Parlement s'adressent au Speaker, Président de la Chambre des communes. C'est donc à retardement que le débat se construit ; les membres du panel ainsi que les députés ne s'interpellent pas directement. Cette procédure d'adresse se retrouve dans certains énoncés tels que "I would like to disagree with the previous and last speaker».

9 Les prérogatives des meneurs des débats dans l'un et l'autre cadre se comparent également dans leurs fonctions de représentation de la Chambre/ de la chaîne, ainsi que dans les fonctions de régulation des débats. 


\subsection{Vers le milieu de l'autre}

10 Politiciens et journalistes passent parfois d'un camp à l'autre. Kilroy, ancien député Labour devenu présentateur éponyme d'un talk-show populaire en est un exemple parmi d'autres. Il déclare au magazine New Woman, à propos des deux fonctions :

There are a lot of similarities. It's just a different platform. I'm a communicator: whether it's teaching, politics or the media, it's all part of the same pattern. The BBC wanted someone who could take on a Cabinet minister, but who could also understand the problems of an unemployed person in Liverpool. An M.P. straddles all those areas. (cité par Livingstone \& Lunt [1994:40])

11 En France, on peut citer le cas de N. Mamère qui a fait le chemin inverse, passant des plateaux des journaux télévisés au Palais Bourbon. Le raisonnement peut s'étendre jusqu'à la rubrique "people» des magazines: nombre de politiciens sont mariés à des journalistes; aux États-Unis, il n'est pas rare de passer du grand écran à de hautes fonctions politiques. Les rapprochements entre politiciens et journalistes ou plus largement les hommes de médias sont parfois liés à la formation commune qu'ils ont reçue. L'ENA et les IEP sont la source d'amitiés de longue date (E. Mougeotte /F. Léotard, J. Chirac / C. Nay). Les grandes universités américaines et les public schools comme Eton favorisent aussi ce type de rencontre.

\subsection{Des instances locutives complexes}

12 Politiciens et journalistes sont des instances locutives complexes. En d'autres termes, il est difficile de savoir « en tant que quoi » les uns et les autres s'expriment; ce sont des porte-parole dont le discours origine est parfois délicat à identifier.

13 Un politicien est tour à tour porte-parole de son parti, du gouvernement au pouvoir, du peuple, des citoyens qu'il administre dans sa circonscription, de ses collègues au Parlement, des gens qui partagent son idéologie... C'est souvent le pronom we qui est responsable de cette ambiguïté. Les quelques exemples donnés ci-dessous illustrent cette multiplicité de voix concomitantes :

14 - Pauline Green est député travailliste membre du Parlement européen, les conservateurs sont alors au pouvoir en Grande-Bretagne :

[1] and part of what we've been doing to date is putting money in aid/ always tied to what we could get out of it in terms of benefit...

[2] and one of the things we need to do.

[3] I think, as a British politician, that we should not be talking to the IRA.

[4] and only by discussions and negotiations will we get that political decision making.

15 Le premier we renvoie sans doute à l'action menée dans le cadre européen; dans l'exemple [3] en revanche, Green se fait porte-parole de toute la classe politique britannique. Le brouillage est de mise; d'autres politiciens de tendance opposée n'opèrent pas différemment :

16 - Kenneth Baker est député conservateur, son parti est au pouvoir, c'est un ancien Ministre :

[5] We've tried, in the last three years under Peter Brooke and now under Patrick

Mayhew to get talks going on again in Northern Ireland.

[6] And we must do everything we can at the Vancouver summit... 
[7] Our society can go on... Considérons maintenant les stratégies langagières plus en profondeur pour identifier des pratiques communes. 


\section{2. À propos de stratégies linguistiques} te politiciens s'éloignent en ce qui concerne les types de discours, le mode énonciatif de l'unicité (le mode du «je ») et l'utilisation d'une stance. En revanche ces communautés tendent à se rapprocher au niveau des catégories des débats, du parallélisme, de l'opposition énonciative ainsi que des stratégies de planification.

\subsection{Types de discours et gestion de l'interaction}

Nous nous intéressons dans un premier temps aux activités co-conduites au niveau des ordres discursifs et des stratégies interactionnelles. Les données considérées à ce niveau permettent de renvoyer au rôle que doivent jouer les deux types de protagonistes au cours de l'interaction médiatique. 


\subsubsection{Les types de discours}

La plupart des types de discours sont sollicités par les deux communautés des journalistes et politiciens, mais dans des proportions bien particulières (voir tableau 1).

Tableau 1. Types de discours

\begin{tabular}{|l|l|l|l|l|l|l|l|l|}
\hline & \multicolumn{2}{l}{\begin{tabular}{l} 
Dimbleby : \\
\multicolumn{1}{l}{116}
\end{tabular}} & \multicolumn{2}{l|}{$\begin{array}{l}\text { Sissons : } \\
\mathbf{5 4}\end{array}$} & \multicolumn{2}{l|}{$\begin{array}{l}\text { Baker : } \\
130\end{array}$} & \multicolumn{2}{l|}{\begin{tabular}{l}
\multicolumn{2}{l|}{107} \\
Typeen discours
\end{tabular}} \\
\hline & Nbre & $\%$ & Nbre & $\%$ & Nbre & $\%$ & Nbre \\
\hline des médias & 76 & 88 & $\mathbf{8 3 , 5}$ & 45 & 5,5 & 7 & 1 & 1 \\
\hline politique & 9,5 & 11 & 5,5 & 3 & $\mathbf{6 1}$ & 80 & $\mathbf{5 1 , 5}$ & 55 \\
\hline vie quotidienne & 13 & 15 & 9,3 & 5 & 15,5 & 20 & 33,6 & 36 \\
\hline institutionnel & 0 & 0 & 2 & 1 & 7 & 9 & 3,5 & 4 \\
\hline propagande & 0 & 0 & 0 & 0 & $\mathbf{1 1}$ & 14 & $\mathbf{8 , 5}$ & 9 \\
\hline économique & 1 & 1 & 0 & 0 & 0 & 0 & 2 & 2 \\
\hline
\end{tabular}

Les politiciens sont dans l'ordre politique (50 et $60 \%$ ) et les journalistes principalement dans l'ordre des médias ( $80 \%$ ). C'est le discours de la vie quotidienne qui est le plus transversal et non pas le discours politique. Nous l'avons vu dans la rubrique précédente, politiciens et journalistes peuvent s'instituer porte-parole des mêmes sources, le citoyen / téléspectateur; ce résultat le montre et en cela les deux communautés sont proches. Elles se distinguent nettement sur d'autres plans, notamment sur la gestion de l'interaction.

\subsubsection{La gestion de l'interaction}

Le journaliste-présentateur a une tâche de plus à accomplir par rapport à un politicien invité : il gère / régule l'interaction. Le tableau ci-dessous le note clairement.

Tableau 2. La gestion de l'interaction

\begin{tabular}{|c|c|c|c|c|c|c|c|c|}
\hline & \multicolumn{2}{|c|}{$\begin{array}{l}\text { Dimbleby : } \\
116\end{array}$} & \multicolumn{2}{|c|}{$\begin{array}{l}\text { Sissons : } \\
54\end{array}$} & \multicolumn{2}{|c|}{$\begin{array}{l}\text { Baker : } \\
130\end{array}$} & \multicolumn{2}{|c|}{$\begin{array}{l}\text { Green : } \\
107\end{array}$} \\
\hline & $\%$ & Nbre & $\%$ & Nbre & $\%$ & Nbre & $\%$ & Nbre \\
\hline Appellatifs & 51,7 & 60 & 48,1 & 26 & 5,4 & 7 & 0,0 & 0 \\
\hline $\begin{array}{l}\text { Gloses } \\
\text { Méta-énonciatives }\end{array}$ & 39,7 & 46 & 20,4 & 11 & 0,0 & 0 & 0,9 & 1 \\
\hline
\end{tabular}


37 Ainsi, les journalistes utilisent des appellatifs pour faire circuler la parole et cela représente entre 48 et $51 \%$ de leur discours; les politiciens oscillent entre 0 et $5 \%$ pour ces marqueurs. De même, les gloses méta-énonciatives (reprises / commentaires du discours de l'autre) représentent 20 à $40 \%$ du discours du journaliste et moins de $1 \%$ du discours d'un politicien.

L'activité de conduite des débats, incombant aux journalistes, les sépare nettement des politiciens.

\subsection{Positionnements énonciatifs}

39 Globalement, les politiciens sont plus investis directement dans leur discours que les journalistes.

\subsubsection{La fracture : unicité et stance}

40 L'unicité (le mode du « je », du « je pense que ») concerne en effet entre 5,5 et 8,5\% du discours des journalistes et entre 28 et $37 \%$ du discours des politiciens sur les deux thèmes.

41 Le recours à l'expression d'une stance directe crée l'écart: le discours directement marqué représente entre 26 et $31 \%$ pour les journalistes et entre 65 et $69 \%$ pour les politiciens ; les énoncés concernés contiennent un modal, un adjectif axiologique, etc.

Les résultats précédents montrent que les proportions sont cohérentes pour les deux journalistes et pour les deux politiciens; cela valide donc, par une analyse microlinguistique, la pertinence du concept de communauté discursive. Les deux journalistes ont la même fonction à remplir et ils le font globalement de façon à peu près similaire, le commentaire est aussi valable pour les politiciens.

\subsubsection{Des territoires communs : débat, parallélisme, opposition}

43 Les deux communautés discursives obtiennent des pourcentages comparables en ce qui concerne le débat (journalistes : $83 \% / 74 \%$; politiciens : 89 et $83 \%$ ). Cela n'est pas surprenant si l'on sait que la macro-catégorie comprend l'activité de débattre et le fait de gérer l'interaction (l'attribution / arrêt des tours de parole...). Il en va de même pour l'énonciation historique (le fait de rappeler des faits objectifs); les pourcentages oscillent entre 11 et $16 \%$.

Comme nous l'avons dit précédemment, le positionnement du parallélisme est adopté par les politiciens et les journalistes, avec parfois la possibilité de représenter les mêmes personnes. Globalement le mode du parallélisme (le fait de parler dans le même sens que d'autres) est activé entre 24 et $29 \%$ pour les journalistes et entre 29,3 et $37,4 \%$ pour les politiciens. Ces chiffres sont largement comparables; les deux communautés tendent donc ici à se rapprocher.

D’ailleurs, le parallélisme exophonique (on parle avec des absents identifiés) obtient le même type de score chez tous les participants : entre 3,7 et 6,2\%.

Cependant, les journalistes représentent les médias et le public sur le plateau, tandis que les politiciens s'instituent porte-parole du peuple en général et de l'institution. 
aient une part aussi importante d'opposition dans leurs discours. En revanche, il est sans doute normal de trouver une part importante d'opposition dans le discours d'un politicien sur un plateau de télévision en présence d'un politicien de tendance opposée. Examinons la réalité linguistique de plus près.

Tableau 3. Débat, parallélisme, opposition

\begin{tabular}{|c|c|c|c|c|c|c|c|c|}
\hline & \multicolumn{2}{|c|}{$\begin{array}{l}\text { Dimbleby : } \\
116\end{array}$} & \multicolumn{2}{|c|}{$\begin{array}{l}\text { Sissons: } \\
54\end{array}$} & \multicolumn{2}{|c|}{$\begin{array}{l}\text { Baker : } \\
130\end{array}$} & \multicolumn{2}{|c|}{$\begin{array}{l}\text { Green : } \\
107\end{array}$} \\
\hline & $\%$ & Nbre & $\%$ & Nbre & $\%$ & Nbre & $\%$ & Nbre \\
\hline Opposition non-marquée & 50 & 56 & 44,5 & 24 & 68 & 88 & 70 & 75 \\
\hline O. diaphonique & 22,5 & 26 & 16,5 & 9 & 5,5 & 7 & 3 & 3 \\
\hline O. exophonique & 3,5 & 4 & 0,0 & 0 & 10,0 & 13 & 10,5 & 11 \\
\hline O. polyphonique & 3,5 & 4 & 5,5 & 3 & 14,5 & 19 & 13 & 14 \\
\hline o. ironique & 1.5 & 2 & 0 & 0 & 0 & 0 & 0 & 0 \\
\hline
\end{tabular}

Si les pourcentages sont du même ordre, on s'aperçoit que les sous-rubriques concernées ne sont pas les mêmes : les journalistes sont clairement diaphoniques et s'opposent à un locuteur sur le plateau, tandis que les politiciens dépassent le cadre de l'interaction interne et sont dans une espèce d'opposition virtuelle.

L'analyse plus précise des énoncés des journalistes révèle que les deux hommes de médias sont loin d'adopter les mêmes stratégies dans la réalité, comme le montrent quelques cas ciblés.

[16] (Panel $\left.{ }^{7}\right)$ What have you done?

[17] (Panel) Where have you gone wrong? 
[18] (Panel) Or when you said you were using clean figures that nobody could argue with, you've excluded that?

- autre thème :

[19] (Panel) Well, Michael Portillo there are two issues, the criticisms of you, your ministers and the ministry of defence for its instinctive defensiveness and insularity/ Ministers for not being pro-active and inquisitive

[20] (Panel) But why haven't they been on this line?

opposition polyphonique

[26] they go out and bomb!

[27] they go out and kill!

[28] No I do not believe the answer lies in internment. (Réponse au journaliste)

60 - opposition exophonique :

[29] The IRA are totally unacceptable in a civilised world

[30] they're murderers!

61 Green :

62 - opposition polyphonique

[31] so the Russian people who have no culture of democracy

[32] that what happened in Warrington was so abhorrent.

63

[33] When they say: we don't want to hurt anybody, we gave a warning

[34] that they will not succeed by these activities

[35] Their community rejects the IRA thugs and terrorists. 

ceux qui pensent que... avec l'appui de ceux qui pensent le contraire. Autrement dit, l'activité d'argumentation pour un politicien est liée à l'opposition et au parallélisme en même temps et sépare l'auditoire en deux franges opposées, a fortiori dans le cadre médiatique. On perçoit très clairement dans les énoncés [26] et [27], que le locuteur se range du côté de ceux qui veulent condamner les membres de l'IRA et ce faisant, se positionne en opposition par rapport à ceux qui soutiennent les indépendantistes. faire un débat stimulant et musclé en traitant d'égal à égal avec le panel. À ce titre, Dimbleby conduit les débats et les initie; Sissons en revanche génère un débat policé où les institutions sont sauvegardées et respectées par sa régulation inhibitrice de l'interaction.

mode de l'opposition permet d'envisager une fracture au sein de la communauté des journalistes vers celle des politiciens pour Dimbleby qui se pose en débatteur et organisateur du débat. On trouve 32 énoncés codés débat + opposition diaphonique chez Dimbleby et à peine $16 \mathrm{chez}$ Sissons. De même 14 énoncés de Dimbleby, contre 7 de Sissons, partagent le trait argumentation.

67 Examinons maintenant le phénomène inverse : le rapprochement que peuvent faire les politiciens vers la communauté des journalistes. Nous allons prendre pour base les stratégies de planification discursive.

\section{3. Éléments de planification discursive}

Le phénomène de planification renvoie globalement à la façon de structurer et d'organiser son discours. Le format académique traditionnel de planification discursive s'apparente à celui d'une pyramide :

One of the occupational hazards of getting a $\mathrm{PhD}$ is a distinct predilection for the traditional pyramid style of exposition. I normally write the way I was trained to write: starting with the foundation and gradually building to the conclusion. Most research papers and engineering reports open with a problem statement, then review the prior art, and move into a detailed discussion of the different options that are considered and the methods that are used. After plowing through twenty pages of basics the patient reader may find a section entitled results with detailed tables, charts, and statistical tests; and after an additional five pages of this, a page or so of conclusions is reached. Phew... (Nielsen 1996)

Une pratique courante concernant la planification du discours dans le cadre médiatique pour les journalistes de presse écrite ou bien plus globalement dans ce que l'on appelle les nouveaux médias (Internet) consiste à retourner la pyramide :

Journalists have long adhered to the inverse approach: start the article by telling the reader the conclusion ("After long debate, the Assembly voted to increase state taxes by 10 percent" follow by the most important supporting information, and end by giving the background. This style is known as the inverted pyramid for the simple reason that it turns the traditional pyramid style around. Inverted-pyramid writing is useful for newspapers because readers can stop at any time and will still get the most important parts of the article. (Nielsen 1996)

Le rôle dialogal du journaliste dans le cadre de notre interaction politique rend difficile les interventions longues et donc l'identification de ce format d'ordre plutôt monologal chez un présentateur de débat qui se doit avant tout de faire parler et de susciter, 
stimuler la parole. Lorsqu'un journaliste de Question Time rappelle des éléments factuels nécessaires à la compréhension du débat, il s'approche du schéma :

This was the result er this is the Law Lords decision today erm that was telling Gloucestershire County Council that they could refuse er care that they had given if they couldn't balance their books and that they were entitled to do that// Anne McElroy

71 Cependant, les interventions longues du journaliste, uniquement celles de Dimbleby d'ailleurs, sont essentiellement des reformulations de la parole ou bien des rappels de faits qui ne sont pas orientés vers une conclusion mais amènent une question, polémique, qui va être redirigée vers un politicien. La structuration de la reformulation est naturellement fidèle à la structuration de l'intervention précédente. L'activité de débat / argumentation pour un journaliste est liée au fait d'introduire une question, pas d'étayer une conclusion, comme le montrent les exemples suivants.

[36] A'right// Well Michael Portillo there are two issues/ the criticisms of you your Ministers and the Ministry of Defence for its instinctive defensiveness and insularity/ Ministers for er not being pro-active and inquisitive/ and then a recommendation that you pay up// What are your answers?

[37] A'right// Michael (applause)/ Michael Por Michael Portillo there've been a number of points made/ the unemployment figures are phoney/ er people are suffering pain despite signs of recovery// What do you say to the gist of what you've heard?

En revanche, il semble que les interventions des politiciens, plus longues et donc plus monologales soient liées au schéma de la pyramide inversée et introduisent une variation intéressante. Nous formulons l'hypothèse que les interventions des politiciens sont majoritairement structurées en double pyramide, ou pyramide redoublée. Pour examiner ce point, regardons une intervention d'un politicien, dont les segments ont été colorisés à partir du système séquences. Ainsi, si notre hypothèse se vérifie, on devrait trouver un segment de type conclusif, lié à l'argumentation, au début et à la fin de l'intervention. Considérons une intervention de Green :

[38] I think we need to understand that what we need is a political solution to the Northern Ireland problem/ And some of my colleagues in the European parliament point to/ for instance/ what Spain has done in the Basque countries where there was also a very strong terrorist movement/ there still is a terrorist movement but it's marginalized/much more less important has very little support amongst the ordinary Basque community and they've done that because they have had a political solution giving autonomy to the Basque people allowing to make their own decision making/ to raise their own taxes/ and they have taken the Basque people with them/ and in so doing have marginalized the terrorists// There is still a small terrorist activity/ but it is much less and it is marginalized/ we need a political solution to the Northern Ireland problem/ and only by discussions and negotiations/ will we get that political decision making //

On voit qu'une forme de conclusion apparaît au début (passage en gras) :

we need a political solution to the Northern Ireland problem.

Suit une analogie, un exemple avec l'Espagne et le problème basque (séquence de description, soulignée). Puis, peu avant la fin de l'intervention, on lit :

we need a political solution to the Northern Ireland problem,

qui est une reprise verbatim du segment présent au début; entre les deux se trouve une argumentation sur les moyens à utiliser : négociation et discussion. 
La structure de l'intervention pourrait donc se matérialiser selon le schéma suivant (figure 1$)^{8}$ :

Figure 1

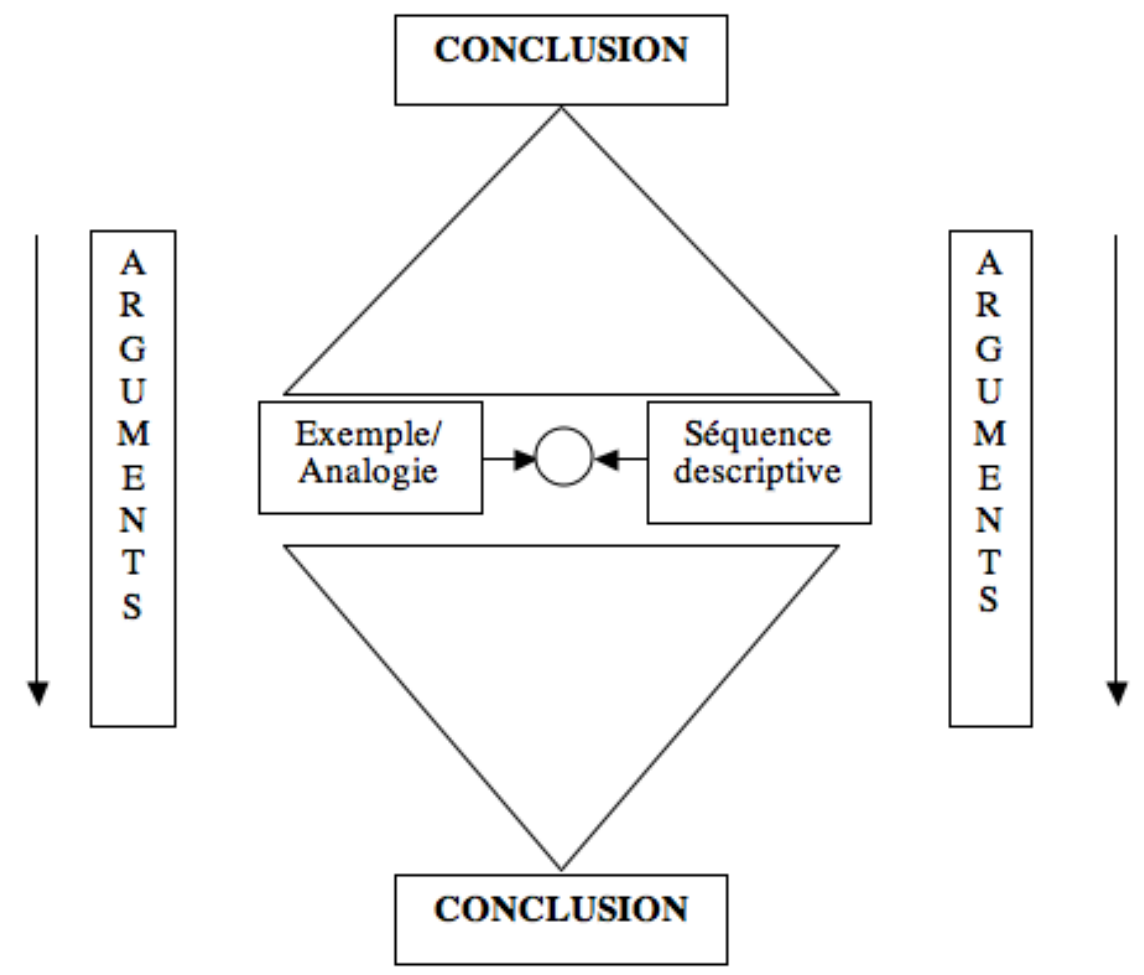

D'autres interventions de Baker semblent répondre au même schéma :

[39] President Yeltsine is the first leader of Russia to be elected by universal suffrage/ and I think that your problem goes much deeper/I think it's the clear advantage of the west that President Yeltsine survives in the course of these next few weeks and months/ and we must do everything we can at the Vancouver summit/ through aid and through assistance/ because if in fact/ Russia collapses into chaos and civil war/ then Yugoslavia/ and that's been in agony/ will be relatively miner/ compared to what could happen in that country//And there would be a massive movement of people west/ int eastern Europe/ into Germany/ it would have a repercussive effect upon the whole stability of Europe// The political stability of Europe/ we've seen what's happened recently with the recent refugee flows into Germany/ that was done on a massive scale// And I know one Russian minister/ coming to a meeting of internal ministers when I was home secretary saying that and this was before the troubles/ he said we reckon seven to ten million Russians who want to leave Russia/ when they get passports/ if in fact chaos and civil war/ overtake Russia/ then there will be tens and tens of millions of people/ with all the consequences of that //We have a very clear interest in the west to ensure that does not happen /

La conclusion énoncée au début :

It is the clear advantage of the west that President Yeltsine survives est reprise indirectement par un énoncé polysémique en fin d'intervention :

we have a very clear interest in the west to ensure that that does not happen.

Cet énoncé concerne plutôt la vague d'immigration qui serait la conséquence d'une fragilisation politique à l'Est. Pourtant, il est impossible de nier que le groupe « in the west 
» fait écho au groupe " of the west » de la conclusion énoncée en ouverture. On remarque ici aussi le fait d'introduire entre les deux conclusions une séquence de description, concernant l'exemple de l'Allemagne.

81 L'effet de ce schéma argumentatif est très fort : il présente l'opinion comme inattaquable grâce à la structure en encadrement. La conclusion se trouve mise en valeur par la répétition et les structures qui l'introduisent vont aussi dans ce sens. "It's the clear advantage that / My view is that " correspondent à des stratégies similaires. Dans le premier cas nous avons affaire à une structure nominalisée extraposée et dans le second à une nominalisation attribut du sujet. Ces configurations syntaxiques permettent toutes d'eux d'évoquer au moins deux fois la proposition. «Advantage » est la première mention (vague), la proposition est l'identification du terme. En prononçant cette séquence, on parle deux fois de la même chose. On peut noter également la présence d'une structure clivée pour introduire la conclusion en ouverture de l'intervention [38] « What we need is... ». Ces structures ont toutes pour fonction essentielle de mettre en valeur les conclusions présentées.

82 En rhétorique, le fait de répéter le même élément en début et en fin de discours prend le nom d'épanadiplose. Cela produit un effet de cycle, qui met en relief le caractère inévitable du contenu. La diversion créée par la séquence descriptive permet d'éviter à l'auditeur de conclure que le locuteur tourne en rond. On voit dans une autre intervention encore plus courte de Baker cet effet de redoublement, cette fois-ci sans exemple. C'est simplement la répétition d'un terme qui permet de mentionner la conclusion une deuxième fois :

well no my view is that you do not speak to the IRA// I think they put themselves beyond the law and also beyond political debate/ That is my view.

La structuration des interventions, pour les politiciens se rapproche donc de ce que l'on peut constater dans les milieux de l'information. On peut sans doute expliquer le redoublement de la conclusion par la nature orale de notre genre de discours: l'immédiateté de la communication télévisuelle pousse sans doute les politiciens à marteler leurs convictions pour ne laisser aucun doute sur ce qu'ils pensent.

\section{Conclusion}

Dans le corpus que nous avons considéré, les deux communautés discursives des journalistes et politiciens sont bien distinctes : elles n'ont pas les mêmes finalités et leur rôle diffère dans ce type d'interaction; cela est très perceptible dans tout ce qui concerne l'aspect méta-interactionnel du discours, dont relèvent les gloses et les types de discours.

En revanche, ces communautés discursives se rapprochent dans l'attitude adoptée par les individus : l'un des journalistes traite d'égal à égal avec les politiciens (en réalisant une forme musclée d'opposition diaphonique contre le panel) et les politiciens font un pas vers les journalistes en adaptant un mode de structuration pratiquée par la communauté discursive des journalistes de presse écrite qui prend alors la forme d'une double pyramide.

86 D'un point de vue variationnel, on pourrait dire qu'un membre d'une communauté donnée se rapproche d'une autre lorsqu'il s'éloigne de la pratique prototypique de la sienne : Dimbleby adopte une attitude très différente de Sissons et en s'éloignant de son confrère, il se rapproche des politiciens. Les différentes communautés discursives 
intervenant dans un même cadre (ici l'interaction médiatique) pourraient donc se situer les unes par rapport aux autres sur un continuum, plutôt que dans des ensembles discrets.

On aboutit donc à la création d'un ordre politico-médiatique hybride, dont les pratiques se nourrissent des différents composants d'origine. Cet ordre ne naît pas simplement de la juxtaposition des domaines / communautés politiques et médiatiques, mais se crée par l'adoption des uns des stratégies classiques de l'autre au cours de l'interaction. Il en résulte que le partage des pratiques transcende les frontières qui bornent les communautés discursives au profit de "communautés de pratique » ou "communautés culturelles " de rang $\mathrm{N}+1$. De telles communautés permettent de rapprocher les types d'acteurs qui partagent, dans leur culture discursive, certaines pratiques langagières, mises en place dans des cadres de communication communs.

\section{BIBLIOGRAPHIE}

Adam, J.M. 1992. Les textes : types et prototypes. Paris : Nathan.

Biber, D. et al. 1999. Longman Grammar of Spoken and Written English. Harlow, GB : Pearson Education Limited.

Ducrot, O. 1984. Le dire et le dit. Paris : Éditions de Minuit.

Dujardin, S. 2001. «L'emphase dans le discours spontané anglais : corrélats acoustiques et prosodiques ». Thèse de Doctorat, sous la direction de D. Hirst, Université d'Aix-en-Provence.

Fairclough, N. 1995. Media Discourse. Londres : Arnold.

Fairclough, N. 1998. « Political discourse in the media: An analytical framework ». In Bell, A. \& P. Garret (dir.), Approaches to Media Discourse. Oxford : Blackwell, 142-162.

Livingstone, S. \& P. Lunt. 1994. Talk on Television. Audience participation and Public Debate. Londres : Routledge.

Nielsen, J. 1996. « Pyramids in cyberspace ». Alertbox, June 1996, <http://www.useit.com>.

O’Donnell, M. 2002. Systemic Code <http://www.wagsoft.com/coder>.

Petit, M. 1998. « Vers une stylistique du hedging : le cas du discours scientifique ». Bulletin de la Société de Stylistique Anglaise 16, 67-80.

Petit, M. 2002. « Termes d'adresses et milieux professionnels », Communication aux journées d'études ASP, EA 2025, décembre 2002.

Rouveyrol, L. 2003. «Étude pragmatique de la variation linguistique dans le débat politique médiatisé en anglais ». Thèse de doctorat sous la direction de F. Dubois-Charlier, Université d'Aix-en-Provence.

Rouveyrol, L. et al. 2005. « A Linguistic toolbox for discourse analysis: Towards a multidimensional handling of verbal interactions ». Discourse Studies 7, 289-313. 
Vion, R. 1998. « La mise en scène énonciative du discours ». In B. Caron (dir.), Proceedings of the 16th International Congress of Linguists [CD Rom]. Oxford : Elsevier Science.

\section{NOTES}

1. Notre traduction.

2. Ce logiciel a été utilisé dans sa version 4.6 de mars 2002. Son concepteur, Mick O’Donnell, le rend disponible gratuitement pour la communauté des linguistes à l'adresse suivante : < www.wagsoft.com/coder>.

3. Cette approche est présentée et illustrée dans Rouveyrol et al. (2005).

4. Pour des raisons d'espace, il n'est pas envisageable de détailler chaque catégorie et de donner une définition pour chaque trait. Nous renvoyons à Rouveyrol (2003: 145-198) pour de plus amples explications.

5. Les catégories mentionnées ci-après ainsi que la distinction genre / type de discours sont empruntées à la Critical Discourse Analysis telle que la pratique Fairclough $(1995,1998)$.

6. Il s'agit là de la théorie séquentielle développée par Adam (1992).

7. L'élément donné entre parenthèses précise quelle est la cible de l'opposition énonciative, contre quel type d'acteur elle se dirige.

8. La matérialisation graphique n'est pas aisée car la prise en compte du sens de discours inverse la dimension. Dans la perspective de Nielsen, la pyramide se construit de bas en haut, des fondations à la conclusion (située sur la pointe). Le discours écrit se matérialise de haut en bas, il en résulte que la première pyramide (droite) est en fait paradoxalement inversée.

\section{RÉSUMÉS}

Cet article propose de s'intéresser à l'interaction intervenant dans un cadre médiatique et plus particulièrement dans le débat Question Time. Les participants au débat forment des communautés discursives bien distinctes : journalistes, hommes politiques ou simples citoyens. Pourtant, si l'on s'intéresse à l'exemple particulier des journalistes et des politiciens, il est possible de déterminer que ces deux communautés tendent à se rapprocher en empruntant les pratiques de l'autre. Après avoir rappelé certaines données concernant l'aspect culturel du débat-panel et les milieux dont proviennent les participants, certaines stratégies langagières, telles que les positionnements énonciatifs et la planification discursive, sont étudiées. L'analyse montre que l'opposition dont font preuve certains journalistes à l'égard des politiciens ainsi que le schéma de la double pyramide que ceux-ci pratiquent rapprochent les deux communautés. L'emprunt et l'adoption des pratiques de l'autre aboutissent à un déplacement des bornes au profit de la création d'une "communauté culturelle» liée à une situation de communication particulière.

This paper focuses on a specific type of mediatized interaction: the panel debate Question Time. Co-participants belong to distinct discourse communities, i.e., they are journalists, politicians or ordinary citizens. However, in the particular example of journalists and politicians, it appears that the two communities start to merge by adopting each other's practices. After looking at the cultural aspect of the panel debate as well as the general backgrounds of the participants, some 
linguistic strategies such as positioning processes and discourse planning are scrutinized. Research indicates that the opposition expressed by certain journalists to the views of politicians, together with the double pyramid pattern used by the latter, draw the two communities closer. The borrowing and adoption of each other's practices blur boundaries and tend to create a "cultural community" identifiable in a given situation of communication.

INDEX

Keywords : cultural community, discourse analysis, double pyramid, journalist, panel debate, professional background, politician

Mots-clés : analyse du discours, communauté culturelle, débat-panel, double pyramide, journaliste, politicien, milieu professionnel

\section{AUTEUR}

\section{LAURENT ROUVEYROL}

Laurent Rouveyrol est maître de conférences à la Maison des langues de l'Université de Nice Sophia-Antipolis. Il appartient à l'UMR 6039, « Bases, Corpus, Langage », où il co-dirige l'opération «Genre de discours et science des textes » au sein de l'équipe de Logométrie. Ses recherches linguistiques se situent dans le champ de l'analyse de discours, l'interaction verbale et portent sur les médias, plus particulièrement les débats télévisés. LauRouveyrol@aol.com 